\title{
EDITORIAL
}

\section{Scientific Knowledge Dissemination and Reproductive Health Promotion in Africa: The Case of AJRH}

\author{
Babatunde Ahonsi \\ Resident Representative, United Nations Population Fund, Beijing, China
}

*For Correspondence: Email: bahonsi@unfpa.org

There are at least three basic reasons for the indispensability of scientific knowledge dissemination to the improvement of reproductive health in Africa. First, Africa is the only major region of the world with extremely heavy burdens of reproductive ill-health, the main causes of which proven low-cost solutions are known but have not been implemented on a massive scale in many of the countries that make up the continent ${ }^{1}$. Second, several key reproductive health $(\mathrm{RH})$ issues including family planning (FP), sexual practices, pregnancy, sexual and gender-based violence, sexuality education, abortion, and infertility have persistently been shaped by widespread misinformation, myths, ignorance and a culture of silence ${ }^{2,3}$. Third, most of the published scientific evidence on a number of critical RH issues in Africa are produced in other geographies and culture areas ${ }^{4,5}$. Thus, their associated recommendations and policy prescriptions are sometimes irrelevant or inappropriate to countries in Africa especially for RH outcomes that are largely determined by sociocultural beliefs, attitudes and practices.

A key need therefore was for regular production and dissemination of rigorous scientific evidence and analysis on Africa, and especially by Africans, on the determinants, correlates and consequences of the major reproductive health challenges confronting Africa. This was and remains the gap that the African Journal of Reproductive Health (AJRH) has been working so hard to narrow in the last 20 years. Simply put, by continuously disseminating African RH research results to many of those who need to have such information, the AJRH has been shaping with scientific evidence, actions in the RH policy, programme development, and service delivery across Africa. It has by so doing added a lot of value to African RH knowledge production since a research is as useful as the sharing of its results with those who need to know.

As a scholar-practitioner in this field, I had the rare privilege, for 11 unbroken years as a Senior Programme Officer with the Ford Foundation (1997-
2008), of working to support the efforts of the Women's Health and Action Research Centre (WHARC) at building the AJRH into a world-class scientific journal. It is thus from my first-hand experience as a witness to the growth of the AJRH that I am able to further outline some of the specific ways the journal has contributed to reproductive health promotion in Africa and some of the critical success factors in this process.

In my considered view, notable among the hitherto often neglected but significant $\mathrm{RH}$ issues in sub-Saharan Africa that the AJRH helped to call needed policy and research attention to over the last twenty years are:

(i) Level and determinants of HIV serodiscordance among married couples

(ii) Etiology of and responses to primary and secondary infertility

(iii) Sexual health-seeking behaviors of unmarried adolescents and young adults and related policy interventions including sexuality education and youth-friendly services

(iv) Multiple dimensions of unmet need for FP, unintended pregnancy, and unsafe abortion

(v) Sociocultural and economic drivers of intergenerational sexual relations

(vi) Biomedicine, prevention and management of reproductive tract infections (and cancers)

(vii) Key populations (including men who have sex with men) in HIV prevention and impact mitigation

(viii) RH needs of persons living with HIV

(ix) Correlates and effective solutions associated with preventable maternal mortality and morbidities

(x) $\quad \mathrm{RH}$ effects of child marriage, female genital mutilation (FGM) and other forms of genderbased violence

As exemplified by many of the papers in this issue of the journal, the range, depth, diversity and cross-cutting nature of the issues that have been addressed by the AJRH since 1997 helped to reaffirm the 
interconnectedness of sexual and reproductive health and rights, and sustainable development, thereby firmly placing the former within the mainstream of public policy discourse across Africa. I strongly believe that it is not a mere coincidence that as the AJRH grew in stature so did we witness a progressive increase in the rate of enactment of strong evidence-based national policies and strategies on critical $\mathrm{RH}$ issues in most African countries ${ }^{7.8}$.

It is to the eternal credit of Professor Friday Okonofua, founder of WHARC, the publisher of the AJRH and his associates and supporters that the AJRH did not suffer premature death or stunted growth. Rather, it successfully navigated several growing pains that made it stronger. Key to this successful evolution of the AJRH into a thriving and credible international scientific journal is a number of factors that deserve to be underlined and these include:

(i) Visionary leadership and astute management of the organization behind the journal

(ii) Agility and adaptability in the face of operational challenges

(iii) Mission fidelity, uncompromising emphasis on quality, and an unwavering Afrocentric-cum-Pan African ethos

(iv) Loyal and sustained partnerships with international, academic, civil society, and funding institutions

(v) Institutional humility and openness to learning and innovation

There are so many other lessons to be learned from the AJRH's story, but suffice to say that the foregoing short summary represents a veritable template for making success of scientific journal publishing in Africa. But it is not a journey for the faint-hearted. To continuously publish four times a year an international standard scientific journal in a very challenging business operating environment like Nigeria, and on a specialized subject matter that is full of controversies and sensitivities, is indeed a very tall order.

Finally, I want to emphasize the huge contribution that the AJRH has made and continues to make to the academic careers of budding African scholars in the fields of public health, gender studies, medicine and allied disciplines. Truth be told, scientific publishing is neither a completely apolitical enterprise nor a level playing field. It is thus often extremely difficult for a young unknown and locally based African scholar to break into a major international journal ${ }^{9}$. We must therefore fully appreciate the role of the AJRH in availing this category of academics the door-opening opportunity to be published on an internationally recognized and respected scientific platform. All those associated with the ARJH are to be saluted for their courage, tenacity, and drive for excellence and are encouraged to stay the course that has led the journal this far.

Nonetheless, the crowded and highly competitive world of scientific publishing offers no guarantees even for a successful journal like the AJRH. So, it is recommended that the ARJH begins to focus more on issues and topics related to improving our understanding of the barriers to effective RH-related policy implementation. It needs to also encourage scholars to investigate more rigorously the factors, processes and outcomes associated with institutional adoption of innovations and individual adoption of new behaviors. For we now have across Africa a largely favorable policy environment and know the solutions to the bulk of the RH challenges still confronting the continent. Africa just needs to seriously get on with universalizing the application of the well-documented proven solutions. The AJRH's continued relevance and sustained value additionally may well derive from giving increasing attention to this cluster of issues in the years to come.

\section{References}

1. Okonofua FE, ed. Confronting the challenge of reproductive health in Africa. 2014; Boca Raton, FA: Brown Walker Press.

2. Blanc AK, Glazer K, Ofomata-Aderemi U and AkinfaderinAgarau F. Myths and misinformation: an analysis of text messages sent to a sexual and reproductive health Q \& A service in Nigeria. Studies in Family Planning 2016; 47:1; 39-53.

3. Robertson L and Szaraz M. The medicalization of FGM. 2016; London: 28 Too Many

4. Santhya KG and Jejeebhoy SJ. Sexual and reproductive health and rights of adolescent girls in low and middle income countries. Global Public Health 2015; 10:2; 189-221.

5. Haberland N and Rogow D. Sexuality education: emerging trends in evidence and practice. Journal of Adolescent Health 2015; 56: S15-S21.

6. World Health Organization. Turning research into practice: suggested actions from case-studies of sexual and reproductive health research. 2006; Geneva: WHO.

7. Papworth E and Nelson N. UNFPA review of policies, strategies and laws related to child marriage, adolescent health, education and employment in five countries in West Africa. 2016; Dakar: UNFPA.

8. United Nations Population Fund. Universal access to reproductive health: progress and challenges. 2016; New York: UNFPA.

9. Zeleza PT. Manufacturing African studies and crises. 1997; Dakar: CODESRIA. 


\title{
EDITORIAUX
}

\section{Diffusion Scientifique des Connaissances et Promotion de Santé de la Reproduction en Afrique: Le Cas de l'AJRH}

\author{
Babatunde Ahonsi \\ Représentant attitré, Fonds des Nations Unies pour la population, Pékin, Chine
}

*Courrier électronique: bahonsi@unfpa.org

Il y a au moins trois raisons fondamentales pour la nécessité de la diffusion de la connaissance scientifique à l'amélioration de santé de la reproduction en Afrique. Tout d'abord, l'Afrique est la seule région majeure du monde avec des charges extrêmement lourdes de santé de la reproduction, dont les principales causes sont connues mais ne sont pas mises en œuvre à grande échelle dans de nombreux pays qui composent Le continent. ${ }^{1}$ Deuxièmement, plusieurs problèmes clés de santé de la reproduction, y compris la planification familiale (PF), les pratiques sexuelles, la grossesse, la violence sexuelle et sexiste, l'éducation sexuelle, l'avortement et la stérilité ont toujours été façonnés par une mauvaise information généralisée, des mythes, de l'ignorance et d'une culture de silence. ${ }^{2,3}$ Troisièmement, la plupart des preuves scientifiques publiées sur un certain nombre de problèmes critiques de SR en Afrique étaient produites dans d'autres régions géographiques et culturelles. ${ }^{4,5}$ Ainsi, leurs recommandations associées et leurs prescriptions politiques étaient parfois sans pertinence ou inappropriées pour les pays d'Afrique, en particulier pour les résultats de SR qui sont en grande partie déterminés par des croyances, des attitudes et des pratiques socioculturelles.

Un besoin essentiel était la production et la diffusion régulières de preuves et d'analyses scientifiques rigoureuses sur l'Afrique, et en particulier par les Africains, sur les déterminants, les corrélations et les conséquences des principaux problèmes de santé de la reproduction auxquels l'Afrique est confrontée. Ce fut et reste l'écart que l'African Journal of Reproductive Health (AJRH) (Revue africaine de santé de la reproduction) a travaillé si fort pour se limiter au cours des 20 dernières années. Autrement dit, en diffusant continuellement les résultats de la recherche sur la SR africaine à bon nombre de ceux qui ont besoin d'avoir de telles informations, l'AJRH a façonné des actions de preuve scientifique dans la politique de la SR,

l'élaboration de programmes et les contextes de prestation de services en Afrique. Ce faisant, elle a ajouté beaucoup de valeur à la production de connaissances en SR africaine, car une recherche est aussi utile que le partage de ses résultats avec ceux qui doivent en connaître. ${ }^{6}$

En tant que praticien scolaire dans ce domaine, j'ai eu le privilège rare pour onze années ininterrompues en tant qu'administrateur principal de programme avec la Fondation Ford (1997-2008) de travailler à soutenir les efforts de WHARC pour construire l'AJRH dans un revue scientifique de classe internationale. C'est ainsi que, grâce à mon expérience de première main, comme témoin de la croissance de l'AJRH, je peux décrire plus en détail certaines des façons spécifiques dont la revue a contribué à la promotion de santé de la reproduction en Afrique et certains des facteurs critiques de succès dans ce processus.

Après mûre réflexion, je pense que parmi les problèmes notables de SR mais jusqu'alors souvent négligés en Afrique subsaharienne, que l'AJRH a aidé à attirer l'attention à la politique et aux recherches nécessaires au cours des vingt dernières années sont les suivants:

(i) Niveau et déterminants de la sérodiffusion du VIH chez les couples mariés

(ii) Étiologie et réponses à l'infertilité primaire et secondaire

(iii) Les comportements sexuels en matière de recherche de la santé chez les adolescents non mariés et les jeunes adultes et les interventions politiques connexes, y compris l'éducation sexuelle et les services adaptés aux jeunes

(iv) Plusieurs dimensions des besoins non satisfaits de PF, des grossesses non désirées et des avortements dangereux

(v) Les facteurs socioculturels et économiques des relations sexuelles intergénérationnelles

(vi) Biomédecine, prévention et gestion des infections de l'appareil de la reproduction (et des cancers)

(vii) Les populations clés (y compris les hommes 
ayant des rapports sexuels avec des hommes) dans la prévention du VIH et l'atténuation de l'impact

(viii) Besoins de la SR des personnes vivant avec le VIH

(ix) Corrélation et solutions efficaces associées à la mortalité et aux morbidités maternelles

(x) Les effets de la SR du mariage des enfants, des MGF et d'autres formes de violence sexiste

La portée, la diversité et la nature transversale des problèmes abordés par l'AJRH depuis 1997 ont contribué à réaffirmer l'interdépendance de santé de la reproduction et les droits sexuels et du développement durable, en situant fermement le premier dans le discours politique public à travers l'Afrique. Je crois forcement que ce n'est pas une simple coïncidence que, à mesure que l'AJRH augmentait de taille, nous avons assisté à une augmentation progressive du taux de promulgation de politiques et de stratégies nationales fondées sur des données probantes sur les problèmes critiques de la SR dans la plupart des pays africains (voir, par exemple, Papworth et Nelson ${ }^{7}$; FNUAP. ${ }^{8}$

C'est à l'éternel crédit du Professeur Friday Okonofua, fondateur de WHARC, l'éditeur de l'AJRH et de ses associés et ses partisans que l'AJRH n'a pas subit de mort prématurée ou de croissance ralentie. Au contraire, il a exploré avec succès plusieurs douleurs croissantes qui l'ont rendu plus fort. La clé de cette évolution réussie de l'AJRH dans une revue scientifique internationale prospère et crédible sont un certain nombre de facteurs qui méritent d'être soulignés:

(i) Direction visionnaire et gestion astucieuse de l'organisation derrière la revue

(ii) L'agilité et l'adaptabilité face aux défis opérationnels

(iii) La fidélité de la mission, l'accent sans compromis sur la qualité et une éthique de l'Acrocentriquecum-Pan africain inébranlables

(iv) Des partenariats loyaux et soutenus avec des universités internationales, des sociétés civiles et des institutions de financement

(v) L'humilité institutionnelle et Ouverture à l'apprentissage et à l'innovation

Il y a tellement d'autres leçons à tirer de l'histoire de l'AJRH, mais il suffit de dire que le court résumé qui précède représente un véritable modèle pour réussir l'édition de revues scientifiques en Afrique. Mais ce n'est pas un voyage pour les malvoyants. Pour publier en permanence quatre fois par an, une revue scientifique standard internationale dans un environnement opérationnel très difficile, comme le Nigéria, et sur un sujet spécialisé qui est plein de controverses et de sensibilités c'est, en effet, beaucoup demander.

Finalement, je veux souligner la contribution considérable que l'AJRH a faite et continue de faire aux carrières académiques des érudits africains en herbe dans les domaines de la santé publique, des études de genre, de la médecine et des disciplines connexes. On dit à la vérité que la publication scientifique n'est ni une entreprise complètement apolitique, ni une égalité des chances. Il est donc extrêmement difficile pour un jeune érudit africain inconnu et localisé de se jeter dans une importante revue internationale. ${ }^{9}$ Nous devons donc apprécier pleinement le rôle de l'AJRH en faisant appel à cette catégorie d'universitaires, la possibilité d'ouvrir la porte à être publiée sur une plate-forme scientifique internationalement reconnue et respectée. Tous ceux qui sont associés à l'ARJH doivent être salués pour leur courage, leur ténacité et leur motivation pour l'excellence et sont encouragés à suivre le cours qui a mené la revue à ce jour. Néanmoins, le monde de l'édition scientifique très fréquenté et hautement compétitif n'offre aucune garantie, même pour une revue réussie comme l'AJRH. Ainsi, il est recommandé que l'ARJH commence à se concentrer davantage sur les questions et les sujets liés à l'amélioration de notre compréhension des obstacles à une mise en œuvre efficace des politiques. Il faut aussi résultats associés à l'adoption institutionnelle des innovations et l'adoption individuelle de nouveaux comportements. Car nous avons maintenant en Afrique un environnement politique largement favorable et nous connaissons les solutions à la majeure partie des défis de la SR auxquels le continent fait face.

L'Afrique doit simplement s'engager dans l'universalisation de l'application des solutions éprouvées bien documentées. La pertinence continue de l'AJRH ainsi que l'ajout de la valeur soutenue peuvent bien découler d'une attention accrue accordée à ce groupe de problèmes dans les années à venir.

\section{Références}

1. Haberland, N. et Rogow, D. Education sexuelle: tendances émergentes en matière de preuve et de pratique. Journal of Adolescent Health 2015; 56: S15-S21.

2. Okonofua, FE, ed. Relever le défi de santé de la reproduction en Afrique. 2014; Boca Raton, FA: BrownWalker Press.

3. Blanc, AK, Glazer, K., Ofomata-Aderemi, U. et AkinfaderinAgarau, F. Mythes et désinformation: une analyse des messages texte envoyés à un service de Q \& A de santé sexuelle et de la reproduction au Nigéria. Studies in Family Planning 2016; 47: 1; 39-53.

4. Santhya, KG et Jejeebhoy, SJ Santé sexuelle et de la rproduction et droits des adolescentes dans les pays à revenu faible et intermédiaire. Global Public Health 2015; 10: 2; 189-221. 
5. Robertson, L et Szaraz, M. La médicalisation de MGF. 2016; Londres: 28 Trop.

6. Organisation mondiale de la santé. Transformer la recherche en pratique: actions suggérées à partir d'études de cas de recherche sur la santé sexuelle et de la reproduction. 2006; Genève: OMS.

7. Papworth, E. et Nelson, N. Examen du FNUAP des politiques, stratégies et lois relatives au mariage des enfants, à la santé des adolescents, à l'éducation et à l'emploi dans cinq pays d'Afrique de l'Ouest. 2016; Dakar: UNFPA.

8. Fonds des Nations Unies pour la population. Accès universel à la santé de la reproduction: progrès et défis. 2016; New York: unfpa.

9. Zeleza, PT. Fabrication d'études et de crises en Afrique. 1997; Dakar: CODESRIA. 